\title{
Compartment Modeling of Dynamic Brain PET - The Impact of Scatter Corrections on Parameter Errors
}

\author{
Ida Häggström ${ }^{\text {a) }}$ \\ Department of Radiation Sciences, Umeå University, Umeå 90187, Sweden \\ C Ross Schmidtlein \\ Department of Medical Physics, Memorial Sloan Kettering Cancer Center, New York 10065, USA \\ Mikael Karlsson and Anne Larsson \\ Department of Radiation Sciences, Umeå University, Umeå 90187, Sweden
}

(Dated: 6 November 2014)

Purpose: The aim of this study was to investigate the effect of scatter and its correction on kinetic parameters in dynamic brain PET tumor imaging. The 2-tissue compartment model was used, and two different reconstruction methods and two scatter correction (SC) schemes were investigated.

Methods: The GATE Monte Carlo (MC) software was used to perform $2 \times 15$ full PET scan simulations of a voxelized head phantom with inserted tumor regions. The two sets of kinetic parameters of all tissues were chosen to represent the 2-tissue compartment model for the tracer FLT, and were denoted $\mathrm{FLT}_{1}$ and $\mathrm{FLT}_{2}$. PET data was reconstructed with both 3D filtered back-projection with reprojection (3DRP) and $3 \mathrm{D}$ ordered-subset expectation maximization (OSEM). Images including true coincidences with attenuation correction (AC) and true+scattered coincidences with $\mathrm{AC}$ and with and without one of two applied SC schemes were reconstructed. Kinetic parameters were estimated by weighted non-linear least squares fitting of image derived time-activity-curves. Calculated parameters were compared to the true input to the MC simulations.

Results: The relative parameter biases for scatter-eliminated data were 15\%, 16\%, 4\%, 30\%, $9 \%$, and $7 \%\left(\mathrm{FLT}_{1}\right)$ and $13 \%, 6 \%, 1 \%, 46 \%, 12 \%$, and $8 \%\left(\mathrm{FLT}_{2}\right)$ for $K_{1}, k_{2}, k_{3}, k_{4}, V_{a}$, and $K_{i}$, respectively. As expected, SC was essential for most parameters since omitting it increased biases by 10 percentage points on average. SC was not found necessary for the estimation of $K_{i}$ and $k_{3}$, however.

There was no significant difference in parameter biases between the two investigated SC schemes or from parameter biases from scatter-eliminated PET data. Furthermore, neither 3DRP nor OSEM yielded the smallest parameter biases consistently, although there was a slight favor for 3DRP which produced less biased $k_{3}$ and $K_{i}$ estimates whilst OSEM resulted in a less biased $V_{a}$. The uncertainty in OSEM parameters was about $26 \%\left(\mathrm{FLT}_{1}\right)$ and $12 \%$ $\left(\mathrm{FLT}_{2}\right)$ larger than for 3DRP although identical postfilters were applied.

Conclusion: SC was important for good parameter estimations. Both investigated SC schemes performed equally well on average and properly corrected for the scattered radiation, without introducing further bias. Furthermore, 3DRP was slightly favorable over OSEM in terms of kinetic parameter biases and SDs.

Keywords: Dynamic PET; Monte Carlo; GATE; compartment modeling; scatter correction

\section{INTRODUCTION}

Quantitative imaging is a field of increasing importance in oncology 1 , including neuro oncology. Dynamic positron emission tomography (PET) offers in vivo information about tumor physiology when observing the time dependent distribution of radiolabeled molecules (tracers) in the patient. There are however many factors that can affect and compromise the quantitative information in PET image sets, which emphasizes the importance to understand and quantify both random and systematic errors in pharmacokinetic model parameters. In order to get proper image values (in units of radioactivity per volume), PET images are normalized and corrected for physical decay, detector dead time, attenuation as well as random and scattered coincidences. The correction for scattered coincidences is of particular importance since up to around $40 \%$ of all registered coincidences of a brain PET scan have undergone single or multiple scattering 2 . Scattering leads to mispositioning of the calculated annihilation site and hence a decrease in image contrast and quantitative accuracy.

The importance of proper corrections for scattered coincidences to improve image quality and quantitation is accepted in the nuclear medicine community. The im- 
pact of scatter and its corrections on the pharmacokinetic parameters is less investigated, however, but there are some studies. For example, Cherry et al.$^{2}$ focused on the impact of scatter corrections (SCs) on pharmacokinetic parameters in brain PET imaging and Wang et al ${ }^{3}$ investigated parameters related to hypoxic tumors in animal PET imaging. These types of studies are usually based on simplified simulations where a scatter distribution (for example Gaussian) is added to the sinogram, or time-activity-curves (TACs) are directly simulated with an added scatter distribution, usually proportional to the total activity ${ }^{214}$. Although these methodologies are often used, they are still relatively severe approximations.

It is difficult if not impossible to evaluate the effect of image reconstruction corrections on pharmacokinetic model parameters using real PET data since the true kinetics is almost always unknown. Furthermore, the origin of each coincidence in real PET data is also unknown, making simulations the only possible way to obtain truly scatter-eliminated data. For a realistic and more comprehensive study of the influence of scattering and its correction, a more advanced simulation method is advantageous. Monte Carlo (MC) simulations offer this extra detailing, with minimal approximations considering the scatter distribution and the ability to know the ground truth regarding kinetic parameter values. Hirano et al.$^{5}$ used a full Monte Carlo simulation to investigate the influence of scatter on kinetic parameters relevant in myocardial PET imaging, but there is still a need to clarify the impact of scatter corrections on kinetic parameter errors and uncertainties in dynamic brain PET.

Within the field of oncology, ${ }^{18} \mathrm{~F}$-labeled 2-deoxy-2$\left({ }^{18} \mathrm{~F}\right)$ fluoro-D-glucose (FDG) is by far the most common PET tracer ${ }^{6 / 7}$. For neuro oncology however, the high uptake of FDG in normal brain tissue (background) is problematic. The tracer 3'deoxy-3'- $\left({ }^{18} \mathrm{~F}\right)$ fluorothymidine (FLT) has shown great potential for imaging tumor proliferation and in later years it has gained ground in the field as a useful radiopharmaceutical for brain tumor imaging $4|6| 8][13$.

The aim of this study was to investigate the impact of scatter and its correction on kinetic parameters for brain FLT PET. For this purpose, we used the GATE MC software to accurately simulate a dynamic, realistic head phantom, followed by image reconstruction with and without scatter correction and finally kinetic parameter estimation based on image derived TACs.

\section{MATERIALS AND METHODS}

\section{II.A. Compartment model}

The pharmacokinetics of FLT is usually described by the 2-tissue compartment model seen in Fig. [1]14]. The model input function, here denoted $C_{p}$, is the timeactivity-curve (TAC) describing the tracer concentration in arterial blood plasma. Similarly, the TACs denoted $C_{1}$ and $C_{2}$ represent the concentrations of free and trapped

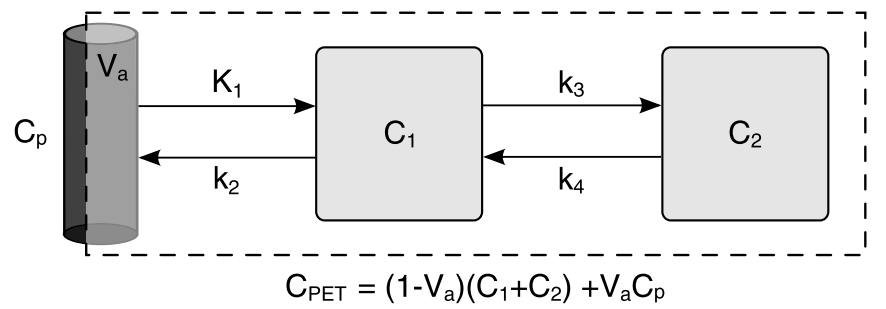

FIG. 1 The 2-tissue compartment model used to describe the pharmacokinetics of e.g. FLT. The model consists of compartments of blood $\left(C_{p}\right)$ and free and bound tracer in tissue $\left(C_{1}\right.$ and $\left.C_{2}\right)$. The model has four rate constants $\left(K_{1}, k_{2}, k_{3}, k_{4}\right)$ governing the transport of tracer, as well as the tissue blood fraction $\left(V_{a}\right) . C_{P E T}$ is the apparent concentration in a PET image VOI or voxel.

(phosphorylated) tracer in tissue, respectively. The rate constants forming the model are the uptake rate from blood to free tracer in tissue, $K_{1}\left(\mathrm{ml} \mathrm{g}^{-1} \mathrm{~min}^{-1}\right)$, the tissue clearance rate, $k_{2}\left(\mathrm{~min}^{-1}\right)$, and the rates of exchange between free and trapped tracer in tissue, $k_{3}\left(\mathrm{~min}^{-1}\right)$ and $k_{4}\left(\mathrm{~min}^{-1}\right)$ respectively. $V_{a}\left(\mathrm{ml} \mathrm{g}^{-1}\right)$ is the fraction of arterial blood appearing in the tissue. For a more detailed description of the model with respect to FLT, see Muzi et al 14 . The macro parameter often referred to as the influx rate constant or the metabolic flux constant, denoted $K_{i}\left(\mathrm{ml} \mathrm{g}^{-1} \mathrm{~min}^{-1}\right)$, is calculated as 9114 .

$$
K_{i}=\frac{K_{1} k_{3}}{k_{2}+k_{3}}
$$

In a PET image, each voxel contains the sum of $C_{1}$ and $C_{2}$, making them impossible to distinguish. The analytical solution for the observed (measured) tissue concentration, $C_{\text {tissue }}$, is ${ }^{15}$ :

$$
\begin{aligned}
C_{\text {tissue }}= & C_{1}+C_{2} \\
= & \frac{K_{1}}{\alpha_{2}-\alpha_{1}}\left[\left(k_{3}+k_{4}-\alpha_{1}\right) e^{-\alpha_{1} t}+\right. \\
& \left.\left(\alpha_{2}-k_{3}-k_{4}\right) e^{-\alpha_{2} t}\right] \otimes C_{p},
\end{aligned}
$$

where " $\otimes$ " denotes temporal convolution and

$$
\alpha_{1,2}=\frac{k_{2}+k_{3}+k_{4}}{2} \mp \frac{\sqrt{\left.\left(k_{2}+k_{3}+k_{4}\right)^{2}-4 k_{2} k_{4}\right)}}{2} \text {. }
$$

Moreover, blood vessels within the voxel or volume-ofinterest (VOI) from where the tissue TAC (TTAC) is derived will contribute to the measured signal. This effect is included in the following way 16 :

$$
C_{P E T}=\left(1-V_{a}\right) C_{\text {tissue }}+V_{a} C_{p} .
$$

\section{II.B. Monte Carlo simulation}

The GEANT4 Application for Tomographic Emission (GATE, v5.0) MC software ${ }^{17}$ was used to perform 15 repetitions of each of two complete dynamic 3D brain PET scans (30 simulations). A previously validated ${ }^{18}$ GE Discovery LS PET camera was simulated, with the following main attributes: 18 detection rings, 672 BGO 
FIG. 2 The voxelized brain phantom used in the simulations, consisting of 9 regions. Each region (except air) was assigned a realistic TAC. Specifically, the blood region was assigned the input function $C_{p}$ and the 14 tu mor regions were each assigned the same TTAC $C_{P E T}$. The spherical blood and tumor regions are labeled with their respective diameter in $\mathrm{mm}$.

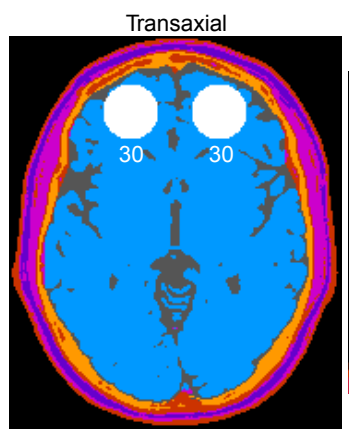

crystals per ring (grand total of 12096 crystals), crystal size $4 \times 8 \times 30 \mathrm{~mm}$, a transaxial field-of-view (FOV) of $550 \mathrm{~mm}$ and an axial FOV of $152 \mathrm{~mm}$. The voxelized BrainWeb head phantom ${ }^{19}$ was used as the digital phantom in GATE. The phantom was segmented into seven materials; air, water, brain tissue, adipose tissue, muscle, skull and general body tissue. Furthermore, seven homogeneous, spherical tumors of diameters $3,6,9,12$, 18, 24 and $30 \mathrm{~mm}$ respectively were distributed in the right hemisphere of the phantom and mirrored to the left hemisphere, resulting in 14 tumors in total. Finally, a blood region in the shape of a $25 \mathrm{~mm}$ diameter sphere was placed centrally in the brain. The size, shape and location of the blood region was not designed to be realistic, but rather to be practical for extraction of an image derived input function of small variance and little influence of partial volume effects (PVEs). The inclusion of a blood region in the head phantom also eliminated the need for an additional simulation of for example the heart region, saving valuable computing time. The phantom had an isotropic voxel size of $1 \times 1 \times 1 \mathrm{~mm}$ and is presented in Fig. 2 .

In order to obtain realistic pharmacokinetics for all normal tissues in the head, smooth fitted TACs from two clinical dynamic brain FLT scans performed at Umeå University Hospital was used for those regions. For both setups, denoted $\mathrm{FLT}_{1}$ and $\mathrm{FLT}_{2}$, the blood region was assigned the input function $C_{p}$ which was generated using Matlab (v.8.1.0, The MathWorks Inc., MA, USA). It had a realistic peak amplitude of $50 \mathrm{kBq} / \mathrm{ml}$ (measured at the University Hospital of Umeå) and a typical input function appearance ${ }^{20}$. For each of the two setups the corresponding tumor TTAC $C_{P E T}$ was also generated using Matlab, according to the 2-tissue model with realistic parameters for gliomas studied with FLT PET ${ }^{911}$. We chose published values for FLT that were as different as possible, to reflect differences in patient kinetics. The values are seen in Table 1

TABLE I Kinetic parameter values for $\mathrm{FLT}_{1}{ }_{11}^{11}$ and $\mathrm{FLT}_{2} \frac{\sqrt{9}}{}$.

\begin{tabular}{lllllll}
\hline & $K_{1}$ & $k_{2}$ & $k_{3}$ & $k_{4}$ & $V_{a}$ & $K_{i}^{*}$ \\
\hline $\mathrm{FLT}_{1}$ & 0.071 & 0.091 & 0.047 & 0.018 & 0.086 & 0.024 \\
$\mathrm{FLT}_{2}$ & 0.111 & 0.131 & 0.017 & 0.012 & 0.122 & 0.013
\end{tabular}

*Values calculated by Eq. 1
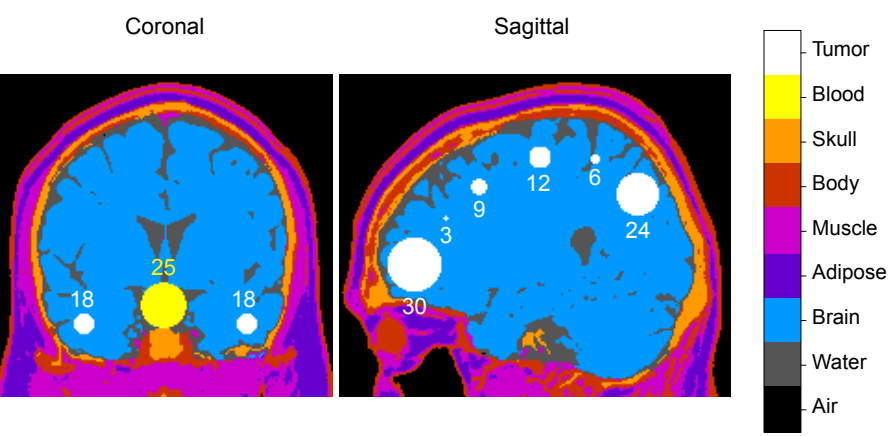

One and the same TTAC $C_{P E T}$ was assigned to all 14 tumor regions, for $\mathrm{FLT}_{1}$ and $\mathrm{FLT}_{2}$, respectively. The simulated TACs for all tissues in the phantom are seen in Fig. 3. The simulated source particles were ${ }^{18} \mathrm{~F}$ positrons with an electron range production cut of $2 \mathrm{~mm}$, a $\delta$-ray production cut of $10 \mathrm{keV}$, and an x-ray production cut of $10 \mathrm{keV} 18 \mid 21$. Physical decay of the sources was turned on with a half-life of $6586.2 \mathrm{~s}$. The activity of each phantom material was read from the generated TACs and updated every second of the simulation for a total PET acquisition time of $60 \mathrm{~min} .15$ simulations of the setup with $\mathrm{FLT}_{1}$ and 15 simulations of the setup with $\mathrm{FLT}_{2}$ were performed (corresponding to 15 scans of each patient) in order to obtain better statistics for the kinetic parameter analysis.

The total number of registered, kept coincidences

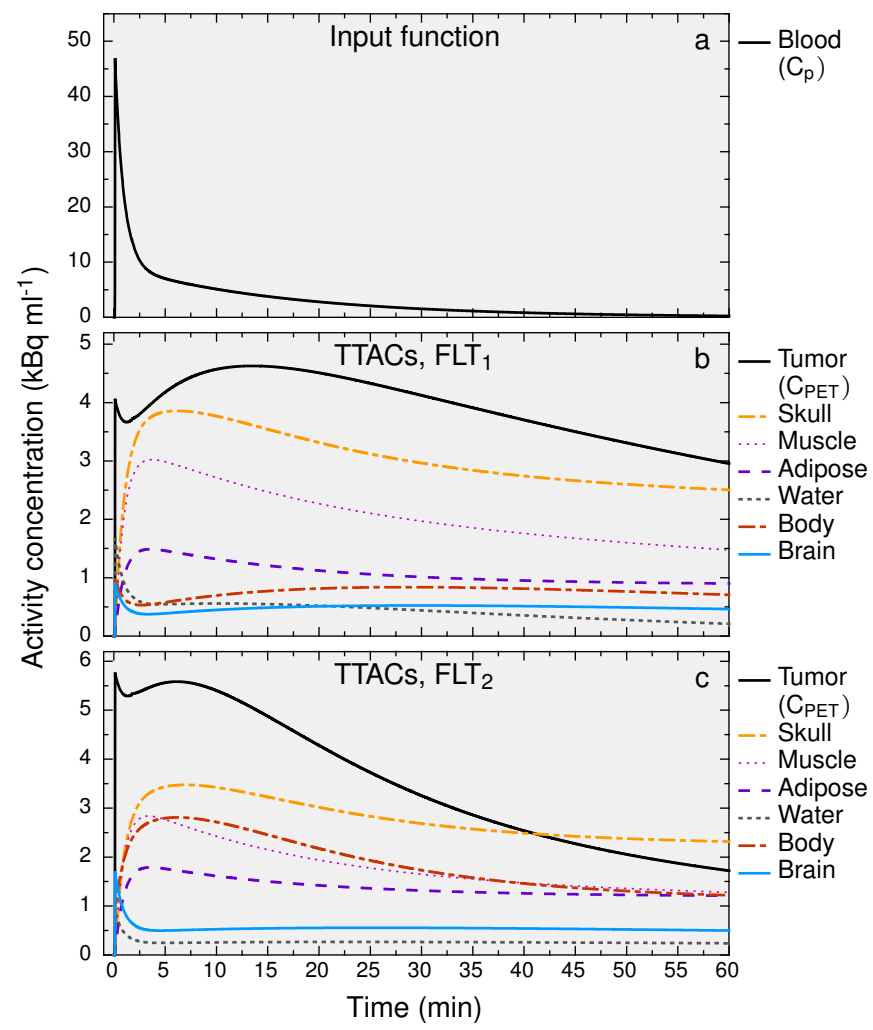

FIG. 3 The generated input function (a) and TTACs for FLT 1 (b) and $\mathrm{FLT}_{2}$ (c) for the tissues in the head phantom, showing the FLT tracer activity over time. 
(true, random and scattered coincidences, referred to as Trues, Randoms and Scatters from now on) in the 15 simulations averaged to $119 \times 10^{6}$ and $137 \times 10^{6}$, with an average scatter fraction (SF) of $28.9 \%$ and $28.4 \%$ for $\mathrm{FLT}_{1}$ and $\mathrm{FLT}_{2}$, respectively. The total coincidence count and countrate in the 28 dynamic frames (see section II.C) ranged from $0-15 \times 10^{6}$ counts and $0-60$ kcps respectively. Note that although simulated, the Randoms were not included in the sinograms used for image reconstruction. The random fraction for the two setups was merely $2 \%$ and the effect of Randoms and their correction was not of interest in this study.

Data from two previous simulations were also used in addition to the 30 main simulations: 1) For normalization correction: A homogeneous, $59 \mathrm{~cm}$ radius cylindrical source without any attenuating medium was simulated with $100 \mathrm{kBq}$ opposite $511 \mathrm{keV}$ photons for $100000 \mathrm{~s}$ without any physical decay. 2) For quantitative calibration: A homogeneous, $150 \mathrm{~mm}$ radius cylindrical source in water was simulated with $1 \mathrm{MBq}{ }^{18} \mathrm{~F}$ positrons for $10000 \mathrm{~s}$ without any physical decay.

Each of the 30 dynamic simulations required about $3300 \mathrm{CPU}$ hours on the computer cluster Akka (Intel Xeon quad-core L5420 CPUs) at the HPC2N collaboration, Umeå University.

\section{II.C. Image reconstruction}

The list-mode Trues and Trues+Scatters were binned into $3 \mathrm{D}$ sinograms $\sqrt{18}$ and reconstructed using two methods: Analytical 3D filtered back-projection with reprojection (3DRP) ${ }^{22}$ and 3D ordered-subset expectation maximization $(\text { OSEM })^{23}$ iterative reconstruction. The image reconstructions were performed using the software STIR (v.2.1) ${ }^{24}$. A Colsher filter with a cut-off frequency of 0.5 pixel $^{-1}$ was applied for 3DRP and 60 sub-iterations with 12 subsets (5 iterations) were used for OSEM. The OSEM settings were chosen to make sure that the tumor VOI values had reached full convergence. Both reconstruction methods and both coincidence count types had normalization, decay and attenuation corrections (AC) applied. Normalization and decay corrections were always present and will not be stated explicitly from now on.

For the AC, the BrainWeb phantom used as input to GATE was converted to a linear attenuation coefficient data map ( $\mu$-map) for $511 \mathrm{keV}$ photons in the respective phantom materials. The normalization sinogram was created from the normalization simulation data which were binned into a sinogram 25 . Both attenuation and normalization data were included in the OSEM loop and used as a pre-correction for 3DRP.

Two different SC schemes were used. The first $\left(\mathrm{SC}_{1}\right)$ was based on the actual Scatters from the simulation and the second $\left(\mathrm{SC}_{2}\right)$ was the SC implemented in STIR, based on the single scatter simulation (SSS) algorithm26|27.

- $\mathbf{S C}_{1}$ : A description of the scheme is found in the appendix. Briefly, the scheme uses the actual Scatters from the GATE simulation and blurs them to create a scatter sinogram estimate, thus preserving the scatter noise in the reconstructed images without the need for an explicit SC in the image reconstruction.

- $\mathbf{S C}_{2}$ : A detailed description of the scatter estimation steps in STIR can be found in the STIR user's guide ${ }^{28}$ and in the paper by Tsoumpas et al. ${ }^{29}$. Briefly, the starting point of the algorithm is the $\mu$ map which is thresholded to obtain scatter points within the imaged object. Using the Klein-Nishina equation, the probability of scatter and detection by a detector pair is calculated for all scatter points, and the sum of all such probabilities forms the scatter estimate. The scheme uses a traditional tail fitting approach, in which the tails of the scatter sinogram is fitted and scaled to counts outside of the imaged object.

Both schemes result in a scatter sinogram estimate. For $3 \mathrm{DRP}$ reconstructions, the scatter estimate was subtracted from the original sinogram before pre-correction for attenuation and normalization. For OSEM reconstructions, the scatter estimate was normalized and attenuation corrected and included as an additive sinogram in the iterative loop. Four combinations of coincidence count type and corrections were reconstructed, with both 3DRP and OSEM:

1) Trues with AC,

2) Trues+Scatters with $\mathrm{ACSC}_{1}$,

3) Trues+Scatters with $\mathrm{ACSC}_{2}$,

4) Trues+Scatters with AC (i.e. omitting SC).

All reconstructed images were postfiltered with a $6.0 \mathrm{~mm}$ FWHM Gaussian transverse filter and a 3-point smoothing filter $\left[\begin{array}{lll}1 & 2 & 1\end{array}\right] / 4$ in the axial direction, in accordance with clinical settings for the GE Discovery LS. The final, reconstructed images were of size $165 \times 165 \times 35$ voxels, with a voxel size of $2 \times 2 \times 4.25 \mathrm{~mm}$.

The dynamic PET data were reconstructed into dynamic image sets with frames at $6 \times 5,3 \times 10,3 \times 20,2 \times 30$, $2 \times 60,2 \times 150,10 \times 300 \mathrm{~s}$, making a total of $60 \mathrm{~min}$.

Finally, a 3DRP reconstruction of the Trues from the calibration simulation was used to create a scale factor to quantitatively calibrate all images from image counts to $\mathrm{Bq} / \mathrm{ml}$.

\section{II.D. Parameter estimation and analysis}

In this study, both the model input function and the tumor TTAC $\left(C_{p}\right.$ and $\left.C_{P E T}\right)$ were extracted from PET image VOIs, i.e. image derived. The input function $C_{p}$ was extracted from a spherical, $25 \mathrm{~mm}$ diameter VOI (488 voxels, $8.3 \mathrm{ml}$ ) covering the complete blood region. Two TTACs were extracted from spherical, $30 \mathrm{~mm}$ diameter VOIs (843 voxels, $14.3 \mathrm{ml}$ ) covering the left and 

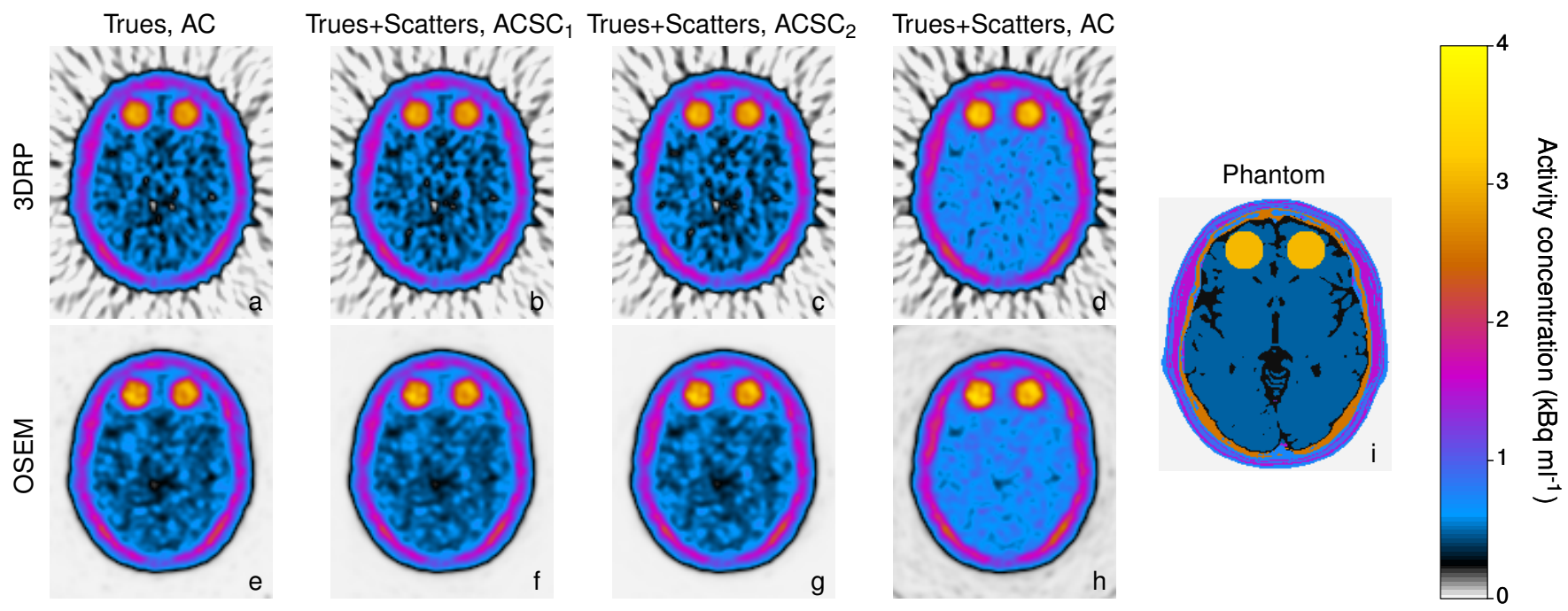

FIG. 4 Example images of the final frame from one of the 15 simulations of $\mathrm{FLT}_{1}$, showing the different corrections, reconstruction methods as well as the real phantom activity image. The coincidence type and correction is indicated on the columns of the figure. AC $=$ attenuation correction, $\mathrm{SC}=$ scatter correction. Top row: 3DRP. Bottom row: OSEM.

right largest tumors, respectively. In order to minimize PVEs in the image derived tumor TTAC, only the two largest $30 \mathrm{~mm}$ tumors were used (the other 12 tumors are intended for another study).

The final result was thus 15 input functions and two TTACs per input function, i.e. a total of 30 tumor TTACs. The recovery coefficient of the image derived TACs was set to unity ${ }^{9}$, i.e. no additional correction for the object size was included to account for PVEs.

Weighted nonlinear least squares (WNLS) was used to fit the dynamic PET data to the compartment mode 14115 . The fitting procedure resulted in estimates of the model parameters $\left[K_{1}, k_{2}, k_{3}, k_{4}, V_{a}\right]$. The sixth kinetic parameter, $K_{i}$, was not fitted but calculated from the other parameters according to Eq. (1). The weight factor of the $\mathrm{i}^{\text {th }}$ frame used in the WNLS fitting was set to a commonly used one, taking radioactive decay and frame length into account 30 :

$$
w_{i}=d_{i} e^{-\lambda t_{i}}
$$

where $d_{i}$ is the frame duration, $\lambda$ the decay constant of ${ }^{18} \mathrm{~F}$ and $t_{i}$ the midtime of the frame. Kinetic parameter estimates were obtained by WNLS fitting of each of the TTACs with the input function as model input, according to Eqs. (2)-(4) with the midtime of each frame used as the time point. The Matlab function lsqnonlin was used for the fitting, and to avoid any effects from initial parameter guesses, the true values were used as initial values.

The estimate of any of the six pharmacokinetic parameter, denoted $\bar{\beta}$, was calculated as the mean of the parameter estimates $\beta_{1}, \beta_{2}, \ldots, \beta_{30}$ of the $30 \mathrm{VOIs}$, resulting in one single set of estimated parameters $\left[K_{1}, k_{2}, k_{3}, k_{4}\right.$, $\left.V_{a}, K_{i}\right]$ for each of the eight coincidence count type, correction and reconstruction method combinations. The (relative) bias in the estimate $\bar{\beta}$ was then calculated as

$$
\left(\bar{\beta}-\beta_{\text {true }}\right) \times \frac{1}{\beta_{\text {true }}} \times 100(\%),
$$

where $\beta_{\text {true }}$ is the true parameter value. The accompanying standard error in the bias was

$$
\frac{s_{\bar{\beta}}}{\sqrt{30}} \times \frac{1}{\beta_{\text {true }}} \times 100(\%)
$$

where $s_{\bar{\beta}}$ is the standard deviation (SD) of parameter estimate $\bar{\beta}$. The relative SD in the parameter estimates was calculated according to

$$
s_{\bar{\beta}} \times \frac{1}{\beta_{\text {true }}} \times 100(\%) .
$$

To determine statistical significance, the calculated biases were analyzed further with 1- and 2-way ANOVA tests, followed by post-hoc Bonferroni corrected pairwise tests to make out individual differences between coincidence count types and corresponding corrections ${ }^{31}$. Results with $\mathrm{p}<0.05$ were considered significant.

Matlab was used for all data fitting and analyses.

\section{RESULTS}

Reconstructions of the final frame of all corrections and both reconstruction methods are seen in Fig. 4. Trues with only AC and Trues+Scatters with AC plus either of the two SCs (columns 1-3 of the figure) resulted in very similar images. Omitting SC (column 4) resulted in an overestimation of the activity concentration, increasing towards the center of the phantom.

Fig. 5 shows representative tumor TTACs and corresponding WNLS fits for one of the 30 available tumor TACs of $\mathrm{FLT}_{1}(15$ simulated PET scans, 2 tumor VOIs each). The cases uncorrected for scatter (Fig. $5 \mathrm{~d}$ and 


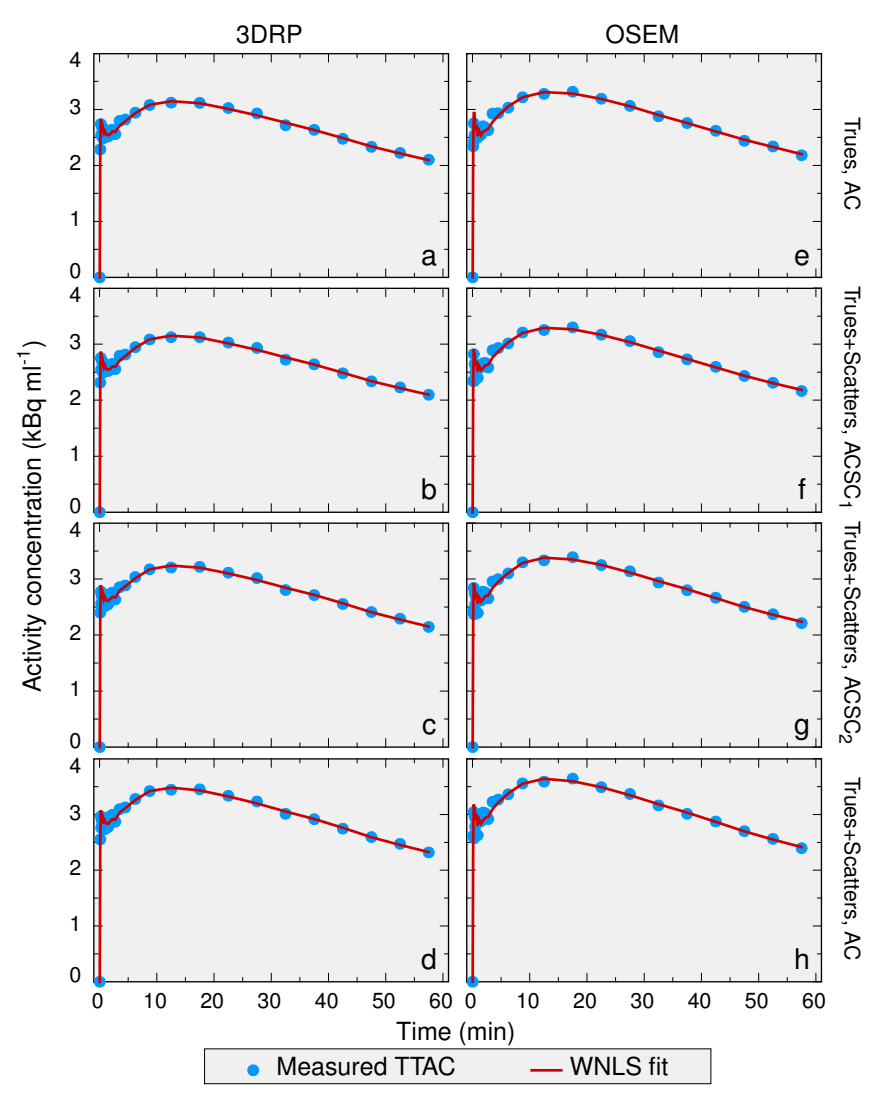

FIG. 5 Representative examples from one of the $30 \mathrm{FLT}_{1}$ tumor VOIs (15 simulations, 2 tumor VOIs each) of both reconstruction methods, showing TTACs and corresponding WNLS fits for the four different combinations of coincidence count type and correction. $\mathrm{AC}=$ attenuation correction, $\mathrm{SC}=$ scatter correction.

h) have roughly $10 \%$ higher VOI values compared to the other three cases.

Values of calculated SD and bias for all six kinetic parameter estimates are seen in Fig. 6. The average parameter biases for Trues with AC, i.e. scatter-eliminated data, were $15 \%, 16 \%, 4 \%, 30 \%, 9 \%$, and $7 \%\left(\mathrm{FLT}_{1}\right)$ and $13 \%, 6 \%, 1 \%, 46 \%, 12 \%$, and $8 \%\left(\mathrm{FLT}_{2}\right)$ for $K_{1}, k_{2}$, $k_{3}, k_{4}, V_{a}$, and $K_{i}$, respectively. There was no significant difference found between the parameter biases for Trues AC, Trues+Scatters $\mathrm{ACSC}_{1}$ and Trues+Scatters $\mathrm{ACSC}_{2}$ for neither 3DRP nor OSEM. Omitting the scatter correction (Trues+Scatters, AC) however resulted in a larger bias for parameters $K_{1}, k_{2}, k_{4}$ and $V_{a}$, as expected. The average difference was about 10 percentage points larger for both $\mathrm{FLT}_{1}$ and $\mathrm{FLT}_{2}$. The difference was not significant for parameters $k_{3}$ and $K_{i}$.

The average bias for 3DRP compared to OSEM was significantly different for three of the six parameters. For $k_{3}$ it was $2 \%$ vs $8 \%\left(\mathrm{FLT}_{1}\right)$ and $0 \%$ vs $4 \%\left(\mathrm{FLT}_{2}\right)$, for $V_{a}$ $18 \%$ vs $3 \%\left(\mathrm{FLT}_{1}\right)$ and $21 \%$ vs $7 \%\left(\mathrm{FLT}_{2}\right)$, and for $K_{i}$ it was $4 \%$ vs $9 \%\left(\mathrm{FLT}_{1}\right)$ and $5 \%$ vs $10 \%\left(\mathrm{FLT}_{2}\right)$, for $3 \mathrm{DRP}$ and OSEM respectively. There was thus no reconstruction method that always kept the bias to a minimum, although 3DRP performed better for two of the three significant parameters, $k_{3}$ and $K_{i}$, and OSEM for one parameter estimation, namely $V_{a}$. Furthermore, the SD (uncertainty) of the parameter estimates was on average $26 \%\left(\mathrm{FLT}_{1}\right)$ and $12 \%\left(\mathrm{FLT}_{2}\right)$ higher for OSEM compared to 3DRP reconstructions.

\section{DISCUSSION}

In this study, we investigated the effect of corrections for scattered coincidences on bias and SD in pharmacokinetic parameter estimates obtained from the 2-tissue compartment model for two typical brain FLT studies. Two different scatter correction schemes were applied and one analytical (3DRP) and one iterative (OSEM) reconstruction method were used.

The MC method was used since it is very difficult to perform repeated dynamic studies like these on a patient or patient-like phantom with known kinetic parameters. Moreover, though simpler simulation methods are possible (e.g. only simulating TACs), those are not as appropriate for an investigation focused on scatter. In this study two sets of kinetic parameter values were simulated, chosen to represent typical brain FLT studies. Furthermore, contrary to simple simulations where the TACs are simulated directly instead of full MC simulations, the scatter fraction is not set (estimated) by the user but is a result based on the shape and size of the object, the distribution of tracer and the camera settings. The magnitude of the SF will affect the scatter corrected images and in turn also the calculation of kinetic parameters. The SF in this study was around $29 \%$ and since it is independent on count rate, simulations with other administered activities would therefore not affect this value but would only affect the image noise level. The image noise level translates to the noise of the VOI, and could in turn affect the WNLS fitting of the image derived TACs to the compartment model. The results obtained in this study are relevant for typical brain FLT PET scans, but for a more general understanding of the impact of scatter corrections of kinetic parameters, a range of administered activities (noise levels) and parameter sets could be simulated.

A bias of zero corresponds to a calculated parameter equal to the true input to GATE. The ideal case with Trues reconstructed with AC can be expected to be bias free, but had biases significantly different from zero. There are many effects that can add to the bias in the estimates. Muzi et al ${ }^{14}$ found the bias of parameters $K_{1}, k_{3}, k_{4}$ and $K_{i}$ to be $-4,26,49$ and $4 \%$, respectively, when applying the 2 -tissue model to simulated tumor TACs under normal conditions, only varying the TAC noise levels. In this study the PVE is likely a large contributor to bias. Furthermore, image reconstruction settings and postfiltering can affect parameter estimate biases. A $6.0 \mathrm{~mm}$ FWHM postfilter was used here, but a postfilter of $3.0 \mathrm{~mm}$ FHWM was also tested for one of the 30 simulations (results not presented). The resulting parameter estimates did not differ from the $6.0 \mathrm{~mm}$ filter why it was kept throughout the study. Other factors 


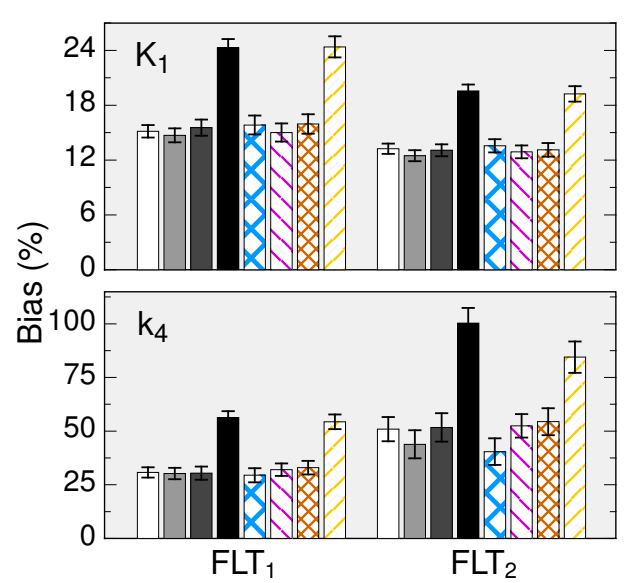

a) Error in kinetic parameter estimates
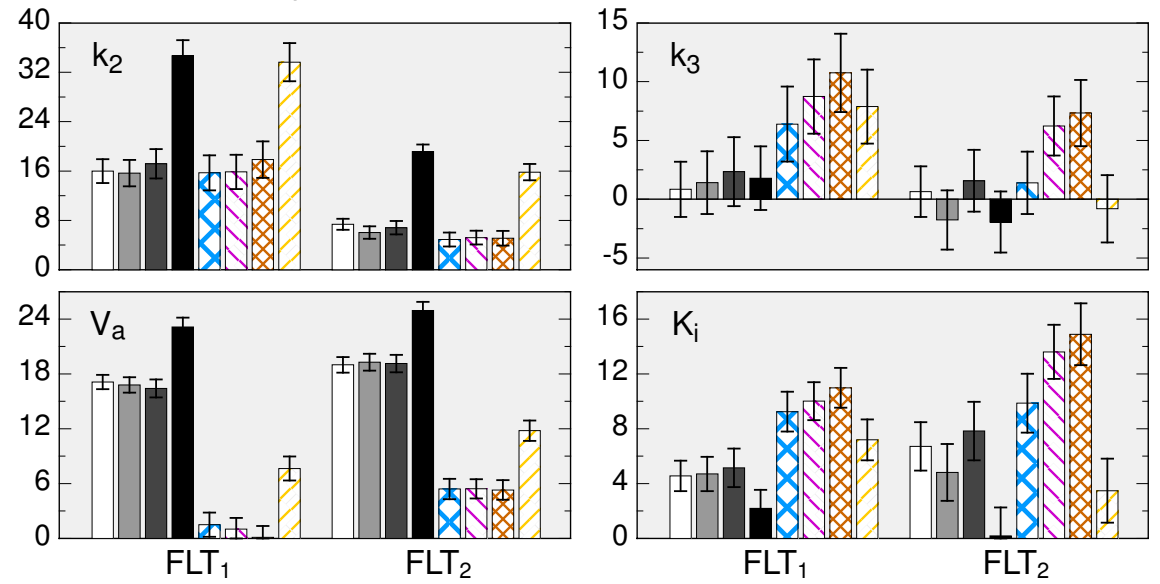

b) Uncertainty in kinetic parameter estimates
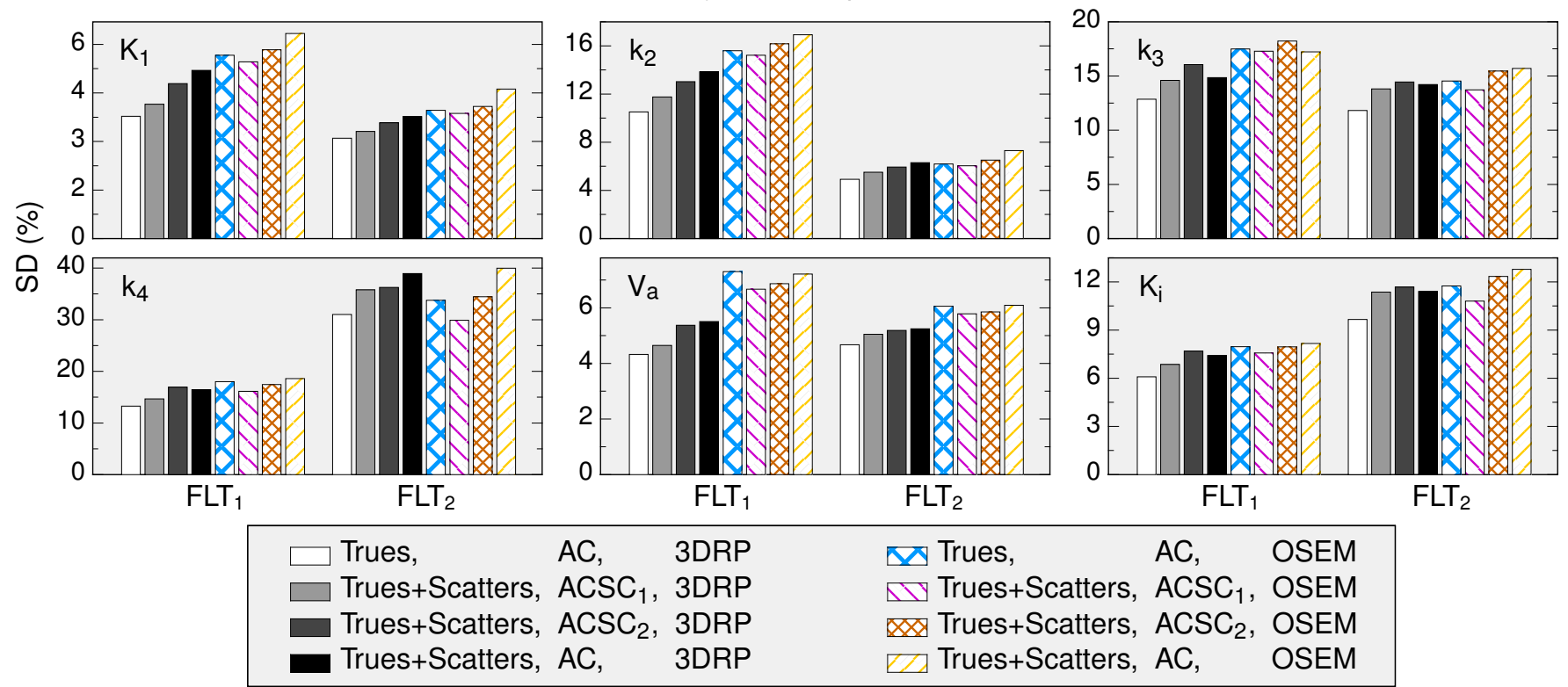

FIG. 6 Relative bias and standard deviation (SD) for all model parameters for the eight combinations of coincidence count type, correction and reconstruction method. The error bars in the bias represent the standard error. Note that the y-axes are scaled differently for better visibility. $\mathrm{AC}=$ attenuation correction, $\mathrm{SC}=$ scatter correction.

that may affect the parameter estimates are the frame sampling 32 , as well as the implementation of the WNLS fitting algorithm and any discrepancies in the calibration constant converting image counts to $\mathrm{Bq} / \mathrm{ml}$. The main focus of this study was not the size of the biases however, but rather the variation between the different coincidence count types, scatter corrections and reconstruction methods.

Trues reconstructed with $\mathrm{AC}$ were used as a reference, and when comparing to Trues+Scatters with $\mathrm{ACSC}_{1}$ and $\mathrm{ACSC}_{2}$, no significant differences were found. Both scatter correction schemes thus worked properly and equally well and did not affect the estimation of the kinetic parameters in any significant manner. Omitting the scatter correction (Trues+Scatters, AC) however resulted in significant differences from Trues with AC, with biases larger by $9,18,25$, and 6 percentage points $\left(\mathrm{FLT}_{1}\right)$ and
$6,11,47$, and 6 percentage points $\left(\mathrm{FLT}_{2}\right)$ for the kinetic parameters $K_{1}, k_{2}, k_{4}$, and $V_{a}$, respectively. Not surprisingly, and in accordance with previous studies 2135 , these results stress the importance of proper SC, not only for direct image analysis, but also for kinetic parameter estimation.

The bias in $k_{3}$ was found independent of SC, implying that the rate of exchange from free tracer to bound tracer in tissue is unaffected by the overestimation of the TACs that comes from not correcting for Scatters. The shape of the TAC stays more or less intact, which is what governs the estimation of $k_{3}$. This agrees with the results found by Cherry et al! ${ }^{2]}$ and Hirano et al ${ }^{5}$. The independence on SC found for $K_{i}$ is not as easily interpreted as it is a macro parameter calculated from three other parameters.

The differences in biases and SDs between $\mathrm{FLT}_{1}$ and 
$\mathrm{FLT}_{2}$ reflect the differences between patients with different kinetics. Both scatter corrections worked equally well on both the slower kinetics described by $\mathrm{FLT}_{1}$ and the faster $\mathrm{FLT}_{2}$, not introducing any parameter biases. Comparing $\mathrm{FLT}_{1}$ and $\mathrm{FLT}_{2}$, the difference in bias was most prominent for $k_{2}$ where the fast kinetics $\left(\mathrm{FLT}_{2}\right)$ resulted in a lower bias and a considerably lower SD.

The difference between OSEM and 3DRP reconstructions was found significant for $k_{3}, V_{a}$ and $K_{i}$. Neither of the reconstruction methods was however always better than the other; $3 \mathrm{DRP}$ performed better for the $k_{3}$ and $K_{i}$ estimation and OSEM for the $V_{a}$ estimation. For mere parameter estimation, 3DRP showed slightly better results compared to OSEM. Furthermore, $K_{i}$ and $k_{3}$ have been found to be important parameters for clinical use ${ }^{910}$. This implies that 3DRP would be preferable to OSEM, with an average bias lower by 7 percentage points for both parameters, for the scatter corrected cases. In accordance with common opinion nonetheless, general image quality of OSEM images is favorable over 3DRP images which include streak artifacts, as is clearly seen in Fig. 4. The poorer image quality of the 3DRP images was not translated to a higher SD of parameter estimates however. On the contrary, 3DRP had about 20\% smaller parameter SDs compared to OSEM. The SD in the tumor VOIs was on average $15 \%$ higher in OSEM than 3DRP images, explaining these results. When studying a VOI in the brain tissue background however, the 3DRP images had a $15 \%$ higher VOI SD compared to OSEM (results not included). These findings are supported by the studies by Barrett et al ${ }^{33}$ and Boellard et al.$^{34}$, where it was found that hot regions in OSEM images have a higher noise level than FBP images and vice versa for cold regions. In the latter study the authors compared the quantitativeness in FBP versus OSEM images in dynamic PET, and found that the estimation of the metabolic rate of glucose (MRGlu) differed for FBP and OSEM imagederived TACs. Consequently, the reconstruction method can obviously be important for some kinetic parameter estimates.

The noise level in OSEM images are largely affected by the number of iterations, and a different setting than the one used here would likely yield slightly different results regarding kinetic parameter SDs. It should be noted again that the same postfilter was used for both reconstruction methods.

The full VOIs used to extract the input function and tumor TTACs were chosen to cover the entire, true regions of the blood and largest tumors, respectively. Although the regions were relatively large $(25 \mathrm{~mm}$ diameter for the blood, $30 \mathrm{~mm}$ diameter for the tumors) thus containing many voxels, voxels close to the edge of the regions will be influenced by PVEs. Furthermore, the recovery coefficient was unity, i.e. no correction for PVEs in relation to object size was included according to common clinical practice. This will effectively lower the apparent VOI activity concentration since the surrounding tissue has lower activity. To further study this effect, the fitting and analysis were also done on parameters calculated from TACs extracted from partial VOIs with an offset by -4 voxels $(8 \mathrm{~mm})$ all around the outer contour of the blood and tumor regions (results not presented). This resulted in calculated biases on average 10 percentage points smaller than for the full VOIs. This difference can be explained by the lesser influence of PVEs on the smaller VOIs. The biases were generally smaller for the partial VOIs, but the uncertainty (SD) was on average $124 \%$ larger (2 percentage points), due to the fewer voxels in the partial VOIs (21 voxels, $0.4 \mathrm{ml}$ and 87 voxels, $1.5 \mathrm{ml}$ for blood and tumors, respectively). The bias trends were similar for the partial VOIs as for the full VOIs, but due to the large uncertainties the statistical significances were lost. Moreover, it is common practice to consider the whole tumor uptake region in PET images, and since the aim of this work did not include consideration of PVEs, and the actual value of the biases was not the main focus, the full VOIs were used.

\section{v. CONCLUSION}

We investigated the impact of scatter and its correction on kinetic parameters from dynamic brain FLT PET, using accurate MC simulations of a PET camera and a realistic head phantom. It comes as no surprise that scatter correction was necessary to avoid bias in most kinetic parameter estimates. The two different scatter correction schemes investigated performed equally well on average and parameters derived from Trues+Scatters with $\mathrm{ACSC}_{1}$ and $\mathrm{ACSC}_{2}$ were not significantly different from the reference scatter eliminated data (Trues with $\mathrm{AC})$. SC was however not found necessary for $K_{i}$ and $k_{3}$ estimation.

Neither of the two reconstruction methods yielded the smallest parameter biases consistently, although 3DRP performed slightly better and resulted in less biased $k_{3}$ and $K_{i}$ estimates. Furthermore, 3DRP resulted in lower parameter uncertainties (SDs), although OSEM images were visually favorable (as expected).

\section{ACKNOWLEDGMENTS}

The authors report no conflicts of interest regarding this work. This work was supported by the Swedish Cancer Society and Lion's Cancer Research Foundation at Umeå University.

\section{Appendix: Scatter correction $\mathrm{SC}_{1}$}

Prior to implementing the STIR scatter correction $\mathrm{SC}_{2}$ we used an idealized scheme on the Monte Carlo data that helped realistically preserve the scatter noise in the final images. This scheme uses the scatter counts from the MC data to estimate the underlying scatter distribution. The scheme is useful when more realistic SC schemes are unavailable. It is an idealized SC scheme since it is based on the actual Scatters, unavailable for 


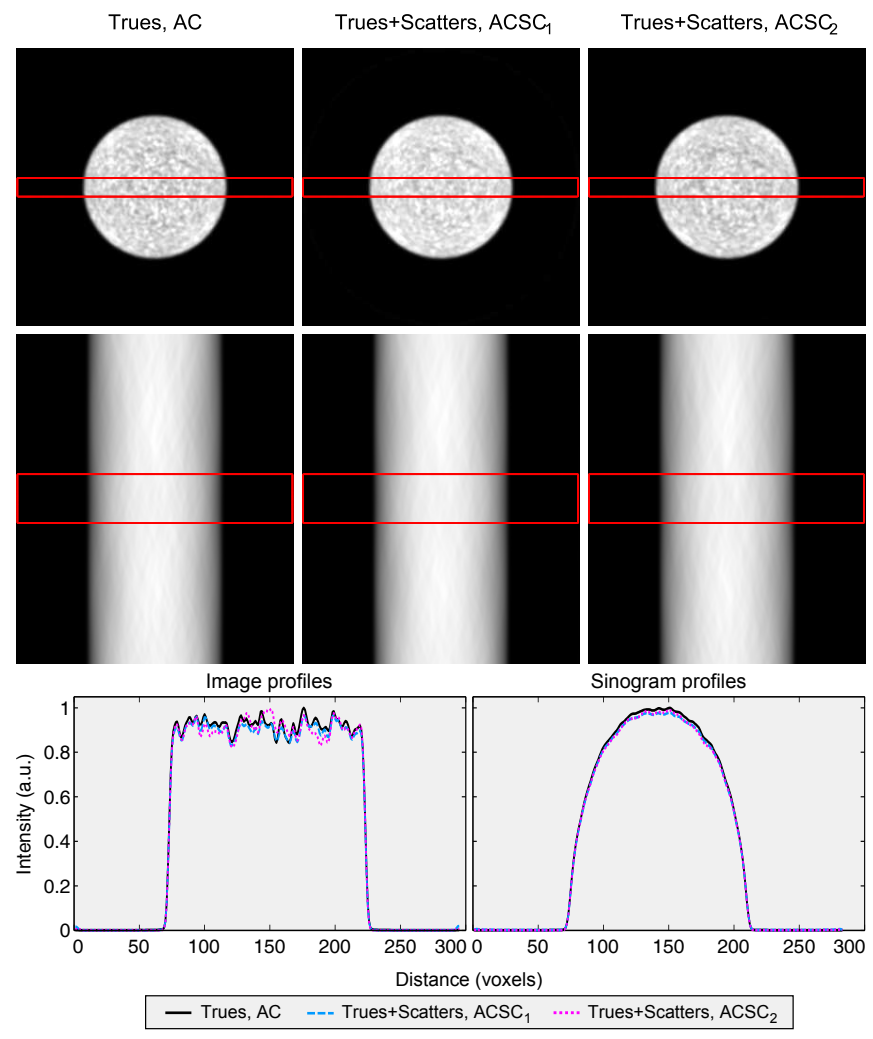

FIG. 7 Reconstructed images (top row) and forward projected images (second row) of a $30 \mathrm{~cm}$ water cylinder with different coincidence type counts and corresponding corrections, together with their profiles (bottom row). $\mathrm{AC}=$ attenuation correction, $\mathrm{SC}=$ scatter correction.

real PET data.

The actual Scatters (from the GATE simulation) are binned and reconstructed with 3DRP. The reconstructed scatter image is smoothed with a $6.0 \mathrm{~mm}$ FWHM Gaussian transverse filter and a 3 -point axial smoothing filter $\left[\begin{array}{lll}1 & 2 & 1\end{array}\right] / 4$ before it is forward projected back into sinogram space. Negative values are truncated from the sinogram, and the sinogram is scaled to the known number of scattered coincidences, resulting in a final scatter sinogram estimate.

OSEM reconstructions (12 subsets, 48 sub-iterations) of a homogeneous, $30 \mathrm{~cm}$ diameter water cylinder are seen in the top row of Fig. 7. The second row shows forward projections of the reconstructed images (sinograms). Three cases are presented in the figure. The first are Trues with $\mathrm{AC}$ (reference), the second implementing the current scheme, $\mathrm{SC}_{1}$, and finally the $\mathrm{SC}$ scheme implemented in STIR, $\mathrm{SC}_{2}$, based on the SSS algorithm.

When studying a large VOI covering the three most central image slices, the resulting $\mathrm{SC}_{1}$ image has a $39 \%$ variance whereas Trues with $\mathrm{AC}$ has $55 \%$ and Trues+Scatters with $\mathrm{ACSC}_{2}$ has $51 \%$. The noise level of the $\mathrm{SC}$ images are thus slightly less than the reference, with $\mathrm{SC}_{1}$ producing the smallest variance. The size of the Gaussian filter, here $6.0 \mathrm{~mm}$ FWHM, affects this result, and more filtering of the $\mathrm{SC}_{1}$ scatter estimate sinogram would produce more noisy scatter corrected images since the smearing would result in some corrected voxels having too many counts subtracted and some voxels too few counts subtracted. Not applying a filter would result in almost identical results as the reference Trues with AC since Scatters would be subtracted almost perfectly.

The current scheme performs well, and compared to the reference Trues with AC and the scheme implemented in STIR, it produces similar images and profiles.

a) Corresponding author: ida.haggstrom@radfys.umu.se

${ }^{1}$ B. F. Kurland, E. R. Gerstner, J. M. Mountz, L. H. Schwartz, C. W. Ryan, M. M. Graham, J. M. Buatti, F. M. Fennessy, E. A. Eikman, V. Kumar, K. M. Forster, R. L. Wahl, F. S, and Lieberman, "QIN. Promise and pitfalls of quantitative imaging in oncology clinical trials," Magn Reson Imaging 30, 1301-12 (2012)

${ }^{2}$ S. R. Cherry and S.-C. Huang, "Effects of scatter on model parameter estimates in 3D PET studies of the human brain," IEEE Trans Nucl Sci 42, 1174-9 (1995)

${ }^{3}$ W. Wang, M. Chen, S. Carlin, C. Oehler, P. Zanzonico, and J. Humm, "Impact ofAttenuation and Scatter Correction in Estimating Tumor Hypoxia-related Kinetic Parameters for FMISO Dynamic Animal-PET Imaging," in IEEE Nucl Sci Symp Conf $\operatorname{Rec}(2008)$ pp. 5234-9.

${ }^{4}$ M. Muzi, A. M. Spence, F. O'Sullivan, D. A. Mankoff, J. M. Wells, J. R. Grierson, J. M. Link, and K. A. Krohn, "Kinetic analysis of 3'-deoxy-3'-18F-fluorothymidine in patients with gliomas." J Nucl Med 47, 1612-21 (2006)

${ }^{5}$ Y. Hirano, K. Koshino, H. Watabe, K. Fukushima, and H. Iida, "Monte Carlo estimation of scatter effects on quantitative myocardial blood flow and perfusable tissue fraction using 3D-PET and (15)O-water," Phys Med Biol 57, 7481-92 (2012).

${ }^{6}$ B. Gulyás and C. Halldin, "New PET radiopharmaceuticals beyond FDG for brain tumor imaging," Q J Nucl Med Mol Imaging 56, 173-90 (2012)

${ }^{7}$ A. F. Shields, J. R. Grierson, B. M. Dohmen, H. J. Machulla, J. C. Stayanoff, J. M. Lawhorn-Crews, J. E. Obradovich, O. Muzik, and T. J. Mangner, "Imaging proliferation in vivo with [F-18]FLT and positron emission tomography," Nat Med 4, 1334-6 (1998)

${ }^{\gamma}$ W. Chen, T. Cloughesy, N. Kamdar, N. Satyamurthy, M. Bergsneider, L. Liau, P. Mischel, J. Czernin, M. E. Phelps, and D. H. S. Silverman, "Imaging proliferation in brain tumors with 18F-FLT PET: comparison with 18F-FDG," J Nucl Med 46, 945-52 (2005)

${ }^{9}$ C. Schiepers, W. Chen, M. Dahlbom, T. Cloughesy, C. K. Hoh, and S.-C. Huang, " $18 \mathrm{~F}$-fluorothymidine kinetics of malignant brain tumors," Eur J Nucl Med Mol Imaging 34, 1003-11 (2007)

${ }^{10}$ A. M. Spence, M. Muzi, J. M. Link, F. O'Sullivan, J. F. Eary, J. M. Hoffman, L. K. Shankar, and K. a. Krohn, "NCI-sponsored trial for the evaluation of safety and preliminary efficacy of 3'deoxy-3'-[18F]fluorothymidine (FLT) as a marker of proliferation in patients with recurrent gliomas: preliminary efficacy studies." Mol imaging Biol 11, 343-55 (2009)

${ }^{11}$ C. Schiepers, M. Dahlbom, W. Chen, T. Cloughesy, J. Czernin, M. E. Phelps, and S.-C. Huang, "Kinetics of 3'-Deoxy-3'18F-Fluorothymidine During Treatment Monitoring of Recurrent High-Grade Glioma," J Nucl Med 51, 720-7 (2010)

12 J. Schwarzenberg, J. Czernin, T. F. Cloughesy, B. M. Ellingson, W. B. Pope, C. Geist, M. Dahlbom, D. H. S. Silverman, N. Satyamurthy, M. E. Phelps, and W. Chen, '3'-deoxy-3'18F-fluorothymidine PET and MRI for early survival predictions in patients with recurrent malignant glioma treated with bevacizumab," J Nucl Med 53, 29-36 (2012)

${ }^{13}$ M. Wardak, C. Schiepers, T. F. Cloughesy, M. Dahlbom, M. E. Phelps, and S.-C. Huang, "18F-FLT and 18F-FDOPA PET kinetics in recurrent brain tumors," Eur J Nucl Med Mol Imaging 41, 1199-209 (2014)

${ }^{14}$ M. Muzi, D. A. Mankoff, J. R. Grierson, J. M. Wells, H. Vesselle, and K. A. Krohn, "Kinetic modeling of 3'-deoxy-3'- 
fluorothymidine in somatic tumors: mathematical studies," J Nucl Med 46, 371-80 (2005)

${ }^{15}$ Y. Ikoma, H. Watabe, M. Shidahara, M. Naganawa, and Y. Kimura, "PET kinetic analysis: error consideration of quantitative analysis in dynamic studies," Ann Nucl Med 22, 1-11 (2008)

${ }^{10}$ M. A. Lodge, R. E. Carson, J. A. Carrasquillo, M. Whatley, S. K. Libutti, and S. L. Bacharach, "Parametric images of blood flow in oncology PET studies using [15O]water," J Nucl Med 41, 1784-92 (2000)

${ }^{17}$ S. Jan, G. Santin, D. Strul, S. Staelens, K. Assié, D. Autret, S. Avner, R. Barbier, M. Bardiès, P. M. Bloomfield, D. Brasse, V. Breton, P. Bruyndonckx, I. Buvat, A. F. Chatziioannou, Y. Choi, Y. H. Chung, C. Comtat, D. Donnarieix, L. Ferrer, S. J. Glick, C. J. Groiselle, D. Guez, P.-F. Honore, S. KerhoasCavata, A. S. Kirov, V. Kohli, M. Koole, M. Krieguer, D. J. van der Laan, F. Lamare, G. Largeron, C. Lartizien, D. Lazaro, M. C. Maas, L. Maigne, F. Mayet, F. Melot, C. Merheb, E. Pennacchio, J. Perez, U. Pietrzyk, F. R. Rannou, M. Rey, D. R. Schaart, C. R. Schmidtlein, L. Simon, T. Y. Song, J.-M. Vieira, D. Visvikis, R. V. de Walle, E. Wieërs, C. Morel, and R. van de Walle, "GATE: a simulation toolkit for PET and SPECT," Phys Med Biol 49, 4543-61 (2004)

${ }^{18}$ C. R. Schmidtlein, A. S. Kirov, S. A. Nehmeh, Y. E. Erdi, J. L. Humm, H. I. Amols, L. M. Bidaut, A. Ganin, C. W. Stearns, D. L. McDaniel, and K. A. Hamacher, "Validation of GATE Monte Carlo simulations of the GE Advance/Discovery LS PET scanners," Med Phys 33, 198-208 (2006)

${ }^{19}$ D. L. Collins, A. P. Zijdenbos, V. Kollokian, J. G. Sled, N. J. Kabani, C. J. Holmes, and A. C. Evans, "Design and Construction of a Realistic Digital Brain Phantom," IEEE Trans Med Imaging 17, 463-468 (1998)

${ }^{20}$ K. B. Contractor, L. M. Kenny, C. R. Coombes, F. E. Turkheimer, E. O. Aboagye, and L. Rosso, "Evaluation of limited blood sampling population input approaches for kinetic quantification of $[18 \mathrm{~F}]$ fluorothymidine PET data," Eur J Nucl Med Mol Imaging 2, 11 (2012)

${ }^{21}$ K. Mitev, G. Gerganov, A. S. Kirov, C. R. Schmidtlein, Y. Madzhunkov, and I. Kawrakow, "Influence of photon energy cuts on PET Monte Carlo simulation results." Med Phys 39, 4175-86 (2012)

${ }^{22}$ P. E. Kinahan and J. G. Rogers, "Analytic 3D Image Reconstruction Using All Detected Events," IEEE Trans Nucl Sci 36,
964-8 (1989)

${ }^{25}$ H. M. Hudson and R. S. Larkin, "Accelerated image reconstruction using ordered subsets of projection data," IEEE Trans Med Imaging 13, 601-9 (1994)

${ }^{24}$ K. Thielemans, C. Tsoumpas, S. Mustafovic, T. Beisel, P. Aguiar, N. Dikaios, and M. W. Jacobson, "STIR: software for tomographic image reconstruction release 2," Phys Med Biol 57, 867$83(2012)$

${ }^{20}$ C. R. Schmidtlein, A. Turner, S. A. Nehmeh, O. Mawlawi, Y. E. Erdi, J. L. Humm, H. I. Amols, and A. Kirov, "SU-FF-I-54: A Monte Carlo Model of the Discovery ST PET Scanner," Med Phys 33, 2009 (2006)

${ }^{20}$ C. C. Watson, D. Newport, and M. E. Casey, "A single scatter simulation technique for scatter correction in 3D PET," in ThreeDim Image Recon Radiol Nucl Med Vol. 4 (1996) pp. 255-68.

${ }^{2}$ C. C. Watson, D. Newport, M. E. Casey, R. A. DeKemp, R. S. Beanlands, and M. Schmand, "Evaluation of Simulation-Based Scatter Correction for 3-D PET Cardiac Imaging," IEEE Trans Nucl Sci 44, 90-97 (1997)

${ }^{28}$ K. Thielemans, D. Sauge, C. Labbé, C. Morel, A. Zverovich, T. Beisel, and C. Tsoumpas, "Software for Tomographic Image Reconstruction User's Guide version 2.1," (2011).

${ }^{29}$ C. Tsoumpas, P. Aguiar, K. S. Nikita, D. Ros, and K. Thielemans, "Evaluation of the Single Scatter Simulation Algorithm Implemented in the STIR Library," in IEEE Nucl Sci Symp Conf Rec, Vol. 00 (2004) pp. 3361-5.

${ }^{30} \mathrm{~F}$. Thiele and R. Buchert, "Evaluation of non-uniform weighting in non-linear regression for pharmacokinetic neuroreceptor modelling," Nucl Med Commun 29, 179-88 (2008)

${ }^{31}$ R. V. Hogg, Engineering Statistics, 1st ed. (Macmillan, New York, 1987).

${ }^{32}$ I. Häggström, A. Larsson, J. Axelsson, A. Garpebring, L. Johansson, C. R. Schmidtlein, J. Sorensen, and M. Karlsson, "The influence of time sampling scheme on kinetic parameters obtained from compartmental modeling of a dynamic PET study - A Monte Carlo study," in IEEE Nucl Sci Symp Med Imaging Conf Rec (2012) pp. 3101-3107.

${ }^{33}$ H. H. Barrett, D. W. Wilson, and B. M. W. Tsui, "Noise properties of the EM algorithm: I. Theory," Phys Med Biol 39, 833-46 (1994)

${ }^{34}$ R. Boellaard, A. van Lingen, and A. A. Lammertsma, "Experimental and clinical evaluation of iterative reconstruction (OSEM) in dynamic PET: quantitative characteristics and effects on kinetic modeling," J Nucl Med 42, 808-17 (2001) 